\title{
MULTIMODALITY IMAGING OF A CARDIAC PSEUDOTUMOR
}

\author{
Danai Kitkungvan, M.D.; Corinne Bontiff, R.C.S.; Michael Reardon, M.D.; Faisal Nabi, M.D. \\ Houston Methodist DeBakey Heart \& Vascular Center, Houston Methodist Hospital, Houston, Texas
}

A 77-year-old asymptomatic female who had recently undergone 2-vessel coronary artery bypass surgery was referred from another hospital for surgical removal of a left atrial mass that was found on a recent transthoracic echocardiogram (TTE).

Cardiac magnetic resonance imaging was obtained for further anatomical evaluation and tissue characterization. Cine-steady state free precession imaging demonstrated a large, wellcircumscribed mass involving the posterolateral mitral annulus without surrounding tissue invasion or compromise to mitral valvular function. T1 turbo spin-echo and T2 turbo spin-echo with fat suppression images showed the mass to be hypointense to myocardium (A and B, arrows), indicating an absence of significant fat or fluid. Absence of resting first-pass perfusion with gadolinium contrast administration indicated a lack of vascularity (C, arrow). Delayed hyperenhancement imaging showed a characteristic pattern of peripheral rim hyperenhancement consistent with a fibrous cap and a dark avascular core (D, arrow). These findings are most consistent with caseous mitral annular calcification. This was then verified with noncontrast cardiac computed tomography that demonstrated a large, hyperdense, calcified mass ( $\mathrm{F}$, arrow).

Once TTE images could be obtained, review of the outside images (apical 4-chamber) similarly demonstrated a large, round, echodense mass at the posterior mitral annulus (E, arrow). Mitral valve function was normal without evidence of either mitral regurgitation or mitral stenosis. The patient was reassured of its benign prognosis, and conservative management/observation was advised.

Keywords: cardiac pseudotumor, left atrial mass

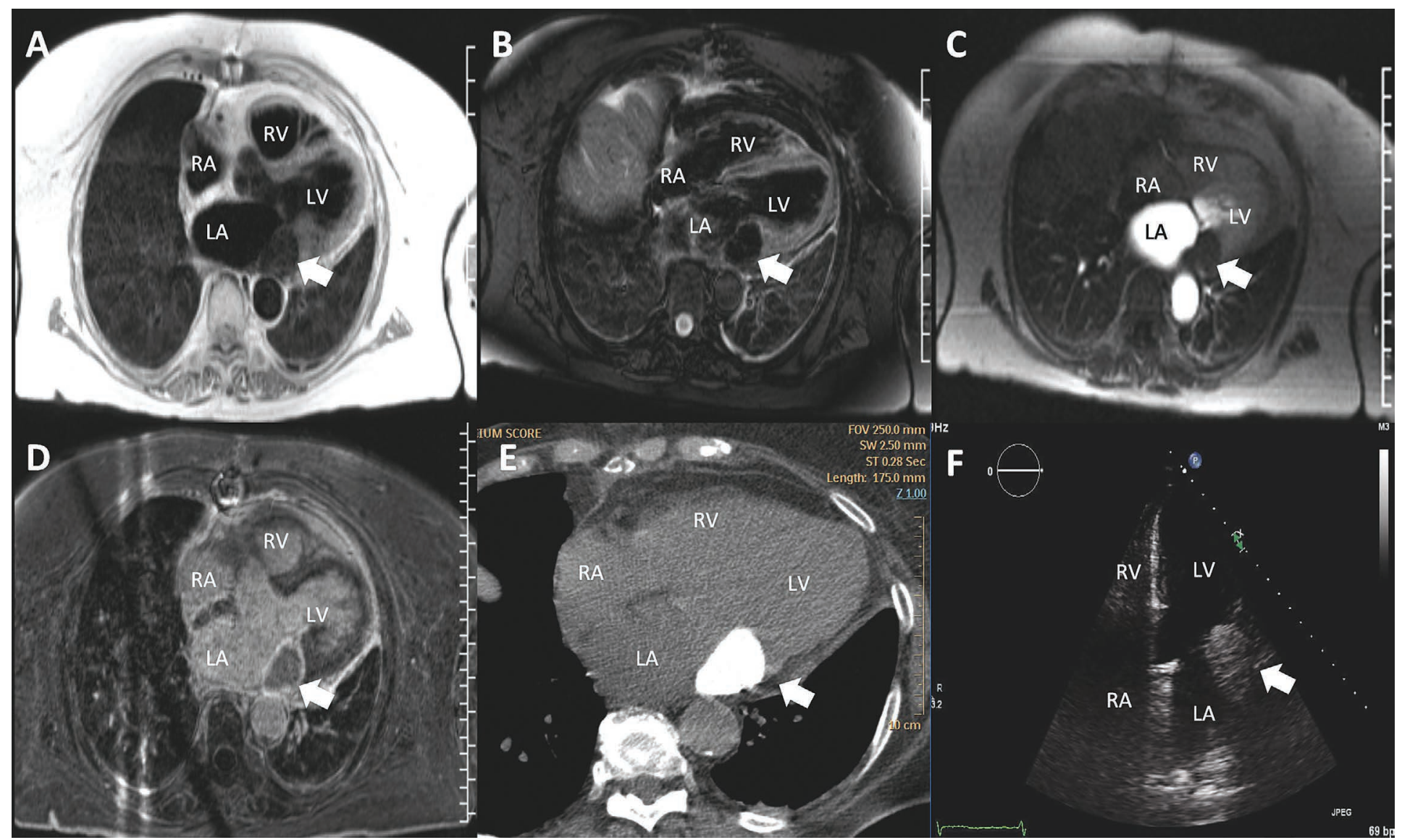

